\title{
Discrete fractional order system vibrations
}

\author{
K.R. (Stevanović) Hedrih, J.A. Tenreiro Machado
}

A B S T R A C T

A theory of free vibrations of discrete fractional order (FO) systems with a finite number of degrees of freedom (dof) is developed. A FO system with a finite number of dof is defined by means of three matrices: mass inertia, system rigidity and FO elements. By adopting a matrix formulation, a mathematical description of FO discrete system free vibrations is determined in the form of coupled fractional order differential equations (FODE). The corresponding solutions in analytical form, for the special case of the matrix of FO properties elements, are determined and expressed as a polynomial series along time. For the eigen characteristic numbers, the system eigen main coordinates and the independent eigen FO modes are determined. A generalized function of visoelastic creep FO dissipation of energy and generalized forces of system with no ideal visoelastic creep FO dissipation of energy for generalized coordinates are formulated. Extended Lagrange FODE of second kind, for FO system dynamics, are also introduced. Two examples of FO chain systems are analyzed and the corresponding eigen characteristic numbers determined. It is shown that the oscillatory phenomena of a FO mechanical chain have analogies to electrical FO circuits. A FO electrical resistor is introduced and its constitutive voltage-current is formulated. Also a function of thermal energy FO dissipation of a FO electrical relation is discussed.

Keywords:

Fractional order element

Generalized function of fractional order

dissipation of system energy

Matrix fractional order differential equation

Eigen fractional order mode

Fractional order oscillator

Mechanical to electrical analogy

\section{Introduction}

Fractional Calculus (FC) represents the generalization of standard differential calculus [1-5] and has been applied in many fields of physics and engineering during the last years [6-11].

In the scientific literature, rheological elements with elastic, viscoelastic and plastic properties were proposed. These elements consist of parallel, series and different hybrid combinations of these simple, mass neglected elements. The fundamentals of these elements are models of the rods with corresponding material properties and axially stressed by pair of opposite forces, with equal intensity. Relations between intensity of the external forces and deformation (extension or compression) of the rods are called constitutive stress strain relations. These constitutive relations are obtained mathematically for complex rheological elements, but, for simple elements, properties are obtained experimentally with ideal elastic materials or ideal viscous fluids. In these models, the rod masses are neglected, as well as the mass inertia, for translation and rotation. In the rheological elements, we can consider neglecting the mass, and to use light standard elements $[12,13]$ with elastic, viscous or plastic properties. Then, for the constitutive relations, the following parameters of the material are adopted: $E$, the coefficient of linear elasticity, $c$, the coefficient of linear rigidity, $\tilde{c}$, the coefficient of cubic non-linearity, $c_{\alpha}$, the coefficient of fractional order (FO) viscoelastic properties (for the limit cases $\alpha=0$ and $\alpha=1$ this parameter, $c_{\alpha=0}$ and $c_{\alpha=1}$, represents the coefficient of elastic and dissipation properties of the material, respectively), $\mu$, the coefficient of viscosity, $n$, the relaxation time, and $\alpha$, the FO coefficient of material dilatation properties. The values of these coefficients can be obtained experimentally.

A standard fractional order element of negligible mass is in the form of an axially stressed rod without bending, that has the ability to resist to deformation under static and dynamic conditions. A standard light FO creep element, for which the constitutive stress-strain relation of the restitution force, as a function of element elongation, is given by expression containing a FO derivative. This constitutive relation is in the form [13-19]:

$P(t)=-\left\{c_{0} x(t)+c_{\alpha} D_{t}^{\alpha}[x(t)]\right\}$

where $D_{t}^{\alpha}[\cdot]$ denotes the fractional operator of $\alpha$ th order with respect to time $t$.

It is known that the Kelvin-Voigt model possesses only one modulus, such that $c_{0}$ is the prolonged (or relaxed, or rubbery) modulus, while $c_{\alpha}=c_{0} \tau_{\alpha}^{\sigma}$, where $\tau_{\alpha}^{\sigma}$ is the retardation (or creep) time, and $0<\alpha<1$ is the order of the fractional derivative [20]. However, here we adopt Riemann-Liouville fractional order derivative. Furthermore, the Kelvin-Voigt model is considered for viscoelastic material, having in 
the parallel coupling between ideal linear elastic and linear viscous (damping) properties. Therefore, we define and we adopt a standard light fractional order element, as coupling element between rigid bodies with translator motion (presenting mass particles).

The following formulation $[15,16,21]$ :

$D_{t}^{\alpha}[x(t)]=\frac{d^{\alpha} x(t)}{d t^{\alpha}}=x^{(\alpha)}(t)=\frac{1}{\Gamma(1-\alpha)} \frac{d}{d t} \int_{0}^{t} \frac{x(\tau)}{(t-\tau)^{\alpha}} d \tau$

where $c$ and $c_{\alpha}$ denote rigidity coefficients (momentary and prolonged), and the order $0<\alpha<1$ is determined experimentally.

Advances in analytical mechanics of FO discrete multi-chain spatial and planar vibrations were developed [12,22]. The discrete FO models of mechanical multi-chain planar systems are considered as possible models of homogeneous deformable body vibrations $[13,14]$. For the case of homogeneous structures (coupled homogeneous chains in which the particles are interconnected by standard light FO elements), FO vibrations of the structure with elongations in parallel directions are analytically considered. We apply the Laplace transform, for solving the governing system of fractional order differential equations (FODE). The corresponding sets of eigen main nets, eigen main chains and eigen like frequency fractional oscillators are identified, and, the analytical expressions are obtained.

Having in mind these ideas the paper is organized as follows. Section 2 describes the discrete system of FO with a finite number of degrees of freedom (dof) with the corresponding matrices of: inertia, linear elastic system rigidity and FO dissipation properties. The generalized function of FO dissipation of the system's total mechanical energy is also introduced. The generalized FO damping force is defined by means of the generalized function of FO energy dissipation. Section 3 discusses the matrix FODE of discrete FO system free vibrations. Extended Lagrange differential equations of second kind in matrix form are derived using kinetic and potential energies and the generalized function of FO energy dissipation. The transformation of matrix FO differential equation using modal matrix and eigen main coordinates of the corresponding linear system is presented. The solution of the matrix FODE for special family of cases with the corresponding theorem is formulated. Section 4 contains solution of matrix FODE for FO chains. Section 5 develops a qualitative and mathematical analogy between mechanical and electrical FO system vibrations. Finally, Section 6 outlines the concluding remarks.

\section{Matrix description of discrete FO system}

We consider a discrete system, with a finite numbers of dof, composed by mass particles and rigid bodies coupled by FO elements of constitutive relation characterized by (1). The number of system's dof is represented by $n$, and the generalized coordinates $\{x\}$ are denoted by a matrix column $\{x\}$ with $n$ elements and generalized coordinates $x_{k}, k=1, \ldots, n$. The system is determined by corresponding mass inertia coefficients $a_{k j}, k, j=1, \ldots, n$, coefficients of elastic properties or rigidities or quasi-elastic properties $c_{k j}, k, j=1, \ldots, n$, and coefficients of FO system properties $c_{\alpha, k j}, k, j=1, \ldots, n$, with $0<\alpha<1$.

Using a matrix formulation of the FO discrete system free vibrations, the following $n \times n$ matrices are determined [23,24]:

- Matrix of coefficients of system mass inertia properties

$$
\mathbf{A}=\left(a_{k j}\right)_{\rightarrow j=1, \ldots, n}^{\downarrow k=1, \ldots, n}
$$

- Matrix of coefficients of system rigidity properties

$$
\mathbf{C}=\left(c_{k j}\right)_{\rightarrow j=1, \ldots, n}^{\downarrow k=1, \ldots, n}
$$

- Matrix of coefficients of system viscoelastic creep FO properties

$$
\mathcal{C}_{\alpha}=\left(c_{\alpha, k j}\right)_{\rightarrow j=1, \ldots, n}^{\downarrow k=1, \ldots, n}
$$

In (5) the symbol $\mathcal{C}_{\alpha}$ denotes the matrix of coefficients of system viscoelastic creep FO, where elements $c_{\alpha, k j}, k, j=1, \ldots, n$, $0<\alpha<1$, are coefficients of FO damping. For $\alpha=0$ they represent the coefficients of elastic rigidity of the system coupling elements between mass particles, while for $\alpha=1$ they are the coefficients of linear damping and system dissipation.

The matrix expressions of discrete FO system kinetic and potential energies are given by

$2 \mathbf{E}_{k}=(\dot{x}) \mathbf{A}\{\dot{x}\}$

$2 \mathbf{E}_{p}=(x) \mathbf{C}\{x\}$

The function of visoelastic creep FO dissipation of system energy, $\alpha \neq 0$, is in the form:

$2 \mathbf{P}_{\alpha \neq 0}=\left(D_{t}^{\alpha}\{x\}\right) \mathbf{C}_{\alpha}\left\{D_{t}^{\alpha}\{x\}\right\}, \quad \alpha \neq 0$

For $\alpha=0$, expression (8) turns into an additional potential energy:

$2 \mathbf{E}_{p, \alpha}=2 \mathbf{P}_{\alpha=0}=(x) \mathbf{C}_{\alpha=0}\{x\}, \quad \alpha=0$

For $\alpha=1$, expression (8) turns into the Rayleigh rheological function of system energy dissipation:

$2 \Phi=2 \mathbf{P}_{\alpha=1}=(\dot{x}) \mathbf{C}_{\alpha=1}\{\dot{x}\}, \quad \alpha=1$

The forces for the generalized coordinates $\{x\}$ are the following:

- Generalized forces of system inertia $\left\{\mathbf{F}_{j}\right\}$ :

$\left\{\mathbf{F}_{j}\right\}=-\left(\frac{d}{d t} \frac{\partial \mathbf{E}_{k}}{\partial\{\dot{x}\}}-\frac{\partial \mathbf{E}_{k}}{\partial\{x\}}\right)=-\mathbf{A}\{\ddot{x}\}$

- Generalized forces $\left\{\mathbf{F}_{c}\right\}$ of system ideal elastic (conservative) properties:

$\left\{\mathbf{F}_{c}\right\}=-\frac{\partial \mathbf{E}_{p}}{\partial\{x\}}=-\mathbf{C}\{x\}$

- Generalized forces $\left\{\mathbf{F}_{\alpha \neq 0}\right\}$ of system with non-ideal visoelastic creep fractional dissipation of system energy:

$$
\left\{\mathbf{F}_{\alpha \neq 0}\right\}=-\frac{\partial \mathbf{P}_{\alpha}}{\partial\left(D_{t}^{\alpha}\{x\}\right)}=-\mathbf{C}_{\alpha}\left\{D_{t}^{\alpha}\{x\}\right\}, \quad \alpha \neq 0
$$

- Generalized forces $\left\{\mathbf{F}_{\alpha}=0\right\}$ of system with additional elastic (conservative) properties for $\alpha=0$ :

$$
\left\{\mathbf{F}_{\alpha=0}\right\}=-\frac{\partial \mathbf{E}_{p, \alpha=0}}{\partial\{x\}}=-\frac{\partial \mathbf{P}_{\alpha=0}}{\partial\{x\}}=-\mathbf{C}_{\alpha=0}\{x t\}, \alpha=0
$$

- Generalized dissipative forces $\left\{\mathbf{F}_{\alpha=1}\right\}$ of system with dissipative (no conservative) properties for $\alpha=1$ :

$$
\left\{\mathbf{F}_{\alpha=1}\right\}=-\frac{\partial \Phi}{\partial\{\dot{x}\}}=-\frac{\partial \mathbf{P}_{\alpha=1}}{\partial\{\dot{x}\}}=-\mathbf{C}_{\alpha=1}\{\dot{x}\}, \alpha=1
$$

\section{Matrix FO differential equation of discrete FO system free vibrations}

In this section the matrix formulation of FODE for FO system's free vibrations and the analytical solutions of some special cases is discussed. In Section 3.1, a generalized FO matrix FODE is presented. The Lagrange FODE of second kind, for FO system dynamics in matrix form, is extended by means of generalized forces of FO dissipation 
expressed by a new generalized function of FO dissipation of total system mechanical energy. For solving matrix FODE, expressed by generalized coordinates of the FO system oscillations, it is useful to start with a coordinate transformation. For that purpose, the generalized coordinates of the corresponding linear system expressed by eigen main coordinates, are included. Section 3.2 studies the solution of linear matrix FODE. Section 3.3 addresses the matrix FODE, expressed by generalized coordinates, using the transformation of generalized into eigen main coordinates of the linear system vibrations. The resulting matrix FODE, with normal coordinates, shows that in an arbitrary case of the matrix of coefficients of system viscoelastic FO dissipative properties along these coordinate, has coupled terms with FO differential operators, and that these modes are not independent. Section 3.4 discusses the special family of the matrix FO system vibrations which posses independent eigen normal fractional modes. For these families, solutions in analytical form are presented and analyzed.

\subsection{Extended Lagrange differential equations of second kind}

The matrix equations of discrete FO free vibrations with a finite number of dof, defined by the column matrix $\{x\}$, are discussed in the sequel.

Principle of dynamic equilibrium of active, fictive and reactive forces:

$\left\{\mathbf{F}_{j}\right\}+\left\{\mathbf{F}_{c}\right\}+\left\{\mathbf{F}_{\alpha}\right\}=\{0\}$

Extended Lagrange FODE of second kind for FO dynamics, using kinetic $\mathbf{E}_{k}$ and potential $\mathbf{E}_{p}$ energies, and generalized $\mathbf{P}_{\alpha}$ of the system's energy fractional order disipation, yields

$\frac{d}{d t} \frac{\partial \mathbf{E}_{k}}{\partial\{\dot{x}\}}-\frac{\partial \mathbf{E}_{k}}{\partial\{x\}}+\frac{\partial \mathbf{E}_{p}}{\partial\{x\}}+\frac{\partial \mathbf{P}_{\alpha}}{\partial\left(D_{t}^{\alpha}\{x\}\right)}+\frac{\partial \Phi}{\partial\{\dot{x}\}}=0$

Independently of the method for obtaining the governing matrix equations of FO system dynamics with finite number of dof:

- Principle of dynamic equilibrium of active, fictive and reactive forces (16). Expressions (7)-(15) of generalized forces of system inertia $\left\{\mathbf{F}_{j}\right\}$ for generalized coordinates $\{x\}$, generalized forces $\left\{\mathbf{F}_{c}\right\}$ of system ideal elastic (conservative) properties for generalized coordinates $\{x\}$ and generalized forces $\left\{\mathbf{F}_{\alpha \neq 0}\right\}$ of system with non-ideal visoelastic creep FO dissipation of system energy for $\alpha \neq 0$, for generalized coordinates $\{x\}$.

- Extended Lagrange FODE of second kind for FO system dynamics (9) using expressions (6)-(10) of kinetic $\mathbf{E}_{k}$ and potential $\mathbf{E}_{p}$ energies and generalized $\mathbf{P}_{\alpha}$ of system energy fractional order disipation. form:

The governing matrix FODE of FO system dynamics is in the

$\mathbf{A}\{\ddot{x}\}+\mathbf{C}_{\alpha}\left\{D_{t}^{\alpha}\{x\}\right\}+\mathbf{C}\{x\}=\{0\}$

The solution of (18), for the case with conventional viscous damping (i.e., $\alpha=1$ ), is obtained via the normal-mode displacement superposition method. This results in the conversion of the $N$ coupled linear damped equations of motion into a set of $N$ uncoupled equations (see, for example, in [25]). This procedure was generalized for fractional derivative damping case (18) in the papers [26,27] using the Rayleigh hypothesis of modal damping.

\subsection{Solution of matrix linear differential equation}

The matrix linear differential equation (LDE) modelling of the linear system dynamics, corresponding to a FODE when rational order terms are omitted, is in the form [28]:

$\mathbf{A}\{\ddot{x}\}+\mathbf{C}\{x\}=\{0\}$

The solution results $[23,19]$ :

$\{x\}=\{A\} \cos (\omega t+\beta)$

where $\{A\}$ denotes a matrix column of amplitudes, $\omega$ represents the frequency and $\beta$ the phase.

The corresponding matrix algebra equations along $\{A\}$ unknown amplitudes yield

$\left(C-\omega^{2} \mathbf{A}\right)\{A\}=\{0\}$

The frequency equation of matrix linear DE is

$f\left(\omega^{2}\right)=\left|C-\omega^{2} \mathbf{A}\right|=0$

Then, the roots of the previous equation are the square of eigen the circular frequencies $\omega_{s}, s=1, \ldots, n$, of linear system dynamics for free vibrations:

Eigen amplitudes of linear system for eigen circular frequencies:

$\frac{A_{k}^{(s)}}{K_{n k}^{(s)}}=C_{s}, \quad s=1, \ldots, n, \quad k=1, \ldots, n$

$A_{k}^{(s)}=C_{s} K_{n k}^{(s)}, \quad s=1, \ldots, n, \quad k=1, \ldots, n$

where

$K_{n k}^{(s)}=K_{n k}\left(\omega_{s}^{2}\right), \quad s=1, \ldots, n, \quad k=1, \ldots, n$

are cofactors of the determinant $\left|C-\omega^{2} \mathbf{A}\right|$ for eigen circular frequencies.

The solution of (19) becomes

$\{x\}=\sum_{s=1}^{s=n}\left\{K_{n k}^{s}\right\} C_{s} \cos \left(\omega_{s} t+\beta_{s}\right)=\mathbf{R}\left\{\xi_{s}\right\}$

The modal matrix $\mathbf{R}$ of linear system of free linear vibrations is

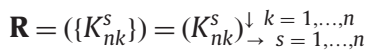

The eigen main coordinate $\xi_{s}$ has the form:

$\left\{\xi_{s}\right\}=\left\{C_{s} \cos \left(\omega_{s} t+\beta_{s}\right)\right\}, \quad s=1, \ldots, n$

where $C_{s}$ and $\beta_{s}, s=1, \ldots, n$, are integral constants defined by initial conditions, initial coordinate and velocities. Then, the generalized coordinate solution of the linear system free linear vibrations is given by

$\{x\}=\sum_{s=1}^{s=n}\left\{K_{n k}^{s}\right\} C_{s} \cos \left(\omega_{s} t+\beta_{s}\right)=\mathbf{R}\left\{\xi_{s}\right\}$

The expression contains also the relation between the generalized coordinates $x_{k}, k=1, \ldots, n$, and the eigen main coordinates $\xi_{s}$, $s=1, \ldots, n$, using modal matrix $\mathbf{R}$ of linear system of free linear vibrations. Introducing in (19) the eigen main coordinates by (28), $\{x\}=\mathbf{R}\left\{\xi_{s}\right\}$, and multiplying by $\mathbf{R}^{\prime}$, the transposed modal matrix, leads to

$\mathcal{A}=\mathbf{R}^{\prime} \mathbf{A} \mathbf{R}=\left(a_{s s}\right)$

$\mathcal{C}=\mathbf{R}^{\prime} C \mathbf{R}=\left(c_{s s}\right)$

Adopting diagonalized matrices, in coordinate system of eigen main coordinates of linear system oscillations, results:

$\mathcal{A}\left\{\ddot{\xi}_{s}\right\}+\mathcal{C}\left\{\xi_{s}\right\}=\{0\}$

This equation represents the matrix linear DE of linear system free vibrations along eigen normal coordinates $\xi_{s}, s=1,2, \ldots, n$, containing system of $n$ independent linear DE, each along only one 
of the eigen main coordinates from set $\xi_{s}, s=1,2, \ldots, n$ :

$a_{s s} \ddot{\xi}_{s}+c_{s s} \xi_{s}=0, \quad s=1, \ldots, n$

where $\omega_{s}^{2}=c_{s S} / a_{s s}$ are squared eigen circular frequencies.

\subsection{Transformation of matrix FO differential equation}

For solving the matrix FODE (18), we use the modal matrix $\mathbf{R}=\left(\left\{K_{n k}^{S}\right\}\right)=\left(K_{n k}^{S}\right)_{\rightarrow S=1, \ldots, n}^{\downarrow k}$, obtained for the matrix linear DE describing free vibrations. It considers as eigen main coordinates $\xi_{s}, s=1, \ldots, n$, for FO system of relation between generalized coordinates $x_{k}, k=1,2, \ldots, n$, and eigen main coordinates $\xi_{s}$, $s=1, \ldots, n$ for the FO system in same form as for the linear system:

$\{x\}=\mathbf{R}\left\{\xi_{s}\right\}$

The previous relation between generalized coordinates and eigen main FO coordinates must satisfy (18). Introducing relation (33) in (18), multiplying by $\mathbf{R}^{\prime}$, the transposed modal matrix, considering (29) and (30) and the relation:

$\mathcal{C}_{\alpha}=\mathbf{R}^{\prime} \mathbf{C}_{\alpha} \mathbf{R}=\left(\mathcal{C}_{(\alpha) s k}\right)_{\rightarrow}^{\downarrow k=1, \ldots, n} \underset{1, \ldots, n}{k=1}$

results into

$\mathcal{A}\left\{\ddot{\xi}_{s}\right\}+\mathcal{C}\left\{\xi_{s}\right\}+\mathcal{C}_{\alpha}\left\{D_{t}^{\alpha}\left\{\xi_{s}\right\}\right\}=\{0\}$

From (35) we conclude that, with the introduction of the eigen main coordinates, $\xi_{s}, s=1, \ldots, n$, in the same form as (33), the two terms $\mathcal{A}\left\{\ddot{\xi}_{s}\right\}$ and $\mathcal{C}\left\{\xi_{s}\right\}$ contain in the main matrix diagonals the elements $a_{s s} \ddot{\xi}_{s}$ and $c_{s s} \xi_{s}, s=1, \ldots, n$, along only one eigen main coordinate $\xi_{s}, s=1, \ldots, n$. The term $\mathcal{C}_{\alpha}\left\{D_{t}^{\alpha}\left\{\xi_{s}\right\}\right\}$, defined by expression (34)-(35), is, in general, not a diagonal matrix along the eigen main coordinates $\xi_{s}, s=1,2, \ldots, n$. In this general case the eigen main coordinates $\xi_{s}, s=1, \ldots, n$, are coupled and the system is not quasilinear FO. Therefore, for each particular case, or family of cases, a method for its solution must be defined.

\subsection{Solution of matrix FO differential equation for a special family} cases

We take into account the special family of the FO system free vibrations for which $\mathcal{C}_{\alpha, S \rightarrow} \downarrow k=1, \ldots, n$, expressed by (34), is diagonal and in the form:

$\mathcal{C}_{\alpha}=\mathbf{R}^{\prime} \mathbf{C}_{\alpha} \mathbf{R}=\left(\mathcal{C}_{(\alpha) s s}\right)$

When (36) is diagonal, Eq. (35) takes the form that all its matrix terms are diagonal. Then, each element on its diagonal is defined along only one of eigen main FO system coordinate $\xi_{s}, s=1, \ldots, n$, and the system of $n$ independent ordinary FODE forms:

$a_{s s} \ddot{\xi}_{s}+c_{(\alpha) s s} D_{t}^{\alpha}\left[\xi_{s}\right]+c_{s s} \xi_{s}=0, \quad s=1, \ldots, n$

For

$\omega_{s}^{2}=\frac{c_{s s}}{a_{s s}}, \quad s=1, \ldots, n$

$\omega_{(\alpha) s}^{2}=\frac{c_{(\alpha) s s}}{a_{s s}}, \quad s=1, \ldots, n$

that is, for the eigen characteristic numbers, expressing the FO properties of the system FO free vibrations of $n$ independent ordinary FODE (37), it is possible to write:

$\ddot{\xi}_{s}+\omega_{(\alpha) s}^{2} D_{t}^{\alpha}\left[\xi_{s}\right]+\omega_{s}^{2} \xi_{s}=0, \quad s=1, \ldots, n$

Each of the ordinary FODE, along only one of eigen main FO system coordinate $\xi_{s}, s=1, \ldots, n$, of system (39), represents the mathematical description of an independent partial FO oscillator with one dof. The corresponding eigen characteristic numbers $\omega_{s}^{2}$ and $\omega_{(\alpha) s}^{2}, s=1, \ldots, n$, express the FO properties of the system free vibrations. The type of each of these FODE was addressed in $[15,23]$. The solutions of each FODE (39) along the eigen main FO system coordinate $\xi_{s}, s=1, \ldots, n$, are possible in the form of a polynomial expansion along time $[29,30]$ :

$$
\begin{aligned}
& \xi_{s}(t)= \xi_{s}(0) \sum_{k=0}^{\infty}(-1)^{k} \omega_{(\alpha) s}^{2 k} t^{2 k} \sum_{j=0}^{k}\left(\begin{array}{l}
k \\
j
\end{array}\right) \frac{(\mp 1)^{j} \omega_{(\alpha) s}^{-2 j} t^{-\alpha j}}{\omega_{s}^{2 j} \Gamma(2 k+1-\alpha j)} \\
&+\dot{\xi}_{s}(0) \sum_{k=0}^{\infty}(-1)^{k} \omega_{(\alpha) s}^{2 k} t^{2 k+1} \sum_{j=0}^{k}\left(\begin{array}{l}
k \\
j
\end{array}\right) \frac{(\mp 1)^{j} \omega_{(\alpha) s}^{-2 j} t^{-\alpha j}}{\omega_{s}^{2 j} \Gamma(2 k+2-\alpha j)}, \\
& s=1, \ldots, n
\end{aligned}
$$

The equation of a fractional derivative Kelvin-Voigt oscillator (39) is presented in the form of a polynomial expansion (40), which may represent an obstacle to mechanical engineers for obtaining a physical interpretation. Analytical solutions could be found in papers [31,32].

On the basis of the analytical approximation of solution of (40), of the FODE (39) for free FO oscillations along each eigen main FO system coordinate $\xi_{s}, s=1, \ldots, n$, is possible to separate two fractional modes $\xi_{s}(t)=\xi_{s}(0) T_{s, \cos }(t, \alpha)+\dot{\xi}_{s}(0) T_{s, \sin }(t, \alpha)$ "similar" to sine $T_{s, \sin }(t, \alpha)$, or to cosine $T_{s, \cos }(t, \alpha), s=1,2, \ldots, n$, with difference in phase as follows $[29,30]$ :

$$
\begin{aligned}
& T_{s, \cos }(t, \alpha)=\sum_{k=0}^{\infty}(-1)^{k} \omega_{(\alpha) s}^{2 k} t^{2 k} \sum_{j=0}^{k}\left(\begin{array}{l}
k \\
j
\end{array}\right) \frac{\omega_{(\alpha) s}^{-2 j} t^{-\alpha j}}{\omega_{s}^{2 j} \Gamma(2 k+1-\alpha j)} \\
& T_{s, \sin }(t, \alpha)=\sum_{k=0}^{\infty}(-1)^{k} \omega_{(\alpha) s}^{2 k} t^{2 k+1} \sum_{j=0}^{k}\left(\begin{array}{l}
k \\
j
\end{array}\right) \frac{\omega_{(\alpha) s}^{-2 j} t^{-\alpha j}}{\omega_{s}^{2 j} \Gamma(2 k+2-\alpha j)}, \\
& s=1, \ldots, n
\end{aligned}
$$

Fig. 1 presents the eigen FO modes $T_{s, \cos }(t, \alpha)$ and $T_{s, \sin }(t, \alpha)$, of the kinetic and FO element parameters used in the discrete system, for the cases: (a) $\omega_{(\alpha) s} / \omega_{s}=1$; (b) $\omega_{(\alpha) s} / \omega_{s}=\frac{1}{3}$; (c) $\omega_{(\alpha) s} / \omega_{s}=3$.

In conclusion we can formulate the following Theorem.

Theorem. Consider the FO dynamical system, with a finite number of dof, defined by $\boldsymbol{A}$, the matrix of coefficients of system inertia properties, $\boldsymbol{C}$, the matrix of coefficients of system rigidity properties and $\mathbf{C}_{\alpha}$, the matrix of coefficients of system viscoelastic creep FO properties. If the modal matrix $\mathbf{R}=\left(\left\{K_{n k}^{S}\right\}\right)=\left(K_{n k}^{S}\right)_{\rightarrow s=1, \ldots, n}^{\downarrow k}$, of the corresponding linear system, produces a diagonalization of matrix $\mathbf{C}_{\alpha}$, of the coefficients of system viscoelastic creep FO properties, using the product $\mathcal{C}_{\alpha}=\mathbf{R}^{\prime} \mathbf{C}_{\alpha} \mathbf{R}=\left(\mathcal{C}_{(\alpha) s s}\right)$, then the system has eigen main independent $F O$ modes $\xi_{s}, s=1,2, \ldots, n$, governed by ordinary FODE: $a_{s s} \ddot{\xi}_{s}+$ $c_{(\alpha) s s} D_{t}^{\alpha}\left[\xi_{s}\right]+c_{s s} \xi_{s}=0$, or eigen normal modes $\zeta_{s}, s=1, \ldots, n$, governed by $\quad \zeta_{s}+\omega_{(\alpha) s}^{2} D_{t}^{\alpha}\left[\zeta_{s}\right]+\omega_{s}^{2} \zeta_{s}=0$, where $\omega_{s}^{2}=c_{s s} / a_{s s}$ and $\omega_{(\alpha) s}^{2}=c_{(\alpha) s s} / a_{s s}$ are two sets of characteristic numbers of FO system oscillations. The first set contains the squared of eigen circular frequencies, the same as for corresponding linear system, and the second contains the characteristic numbers, expressing FO system properties.

\section{Solution of matrix FO differential equation for a FO chain}

Many examples of real FO system free vibrations are possible to be described with a model in which (34) is diagonal. Therefore, finding the solution of this family of matrix FODE is important for applications in the analysis of system dynamics. This case appears when the relation between matrix (4) of coefficients system rigidity properties $C=\left(c_{k j}\right)_{\rightarrow j=1, \ldots, n}^{\downarrow k=1, \ldots, n}$ and matrix (5) of coefficients of system viscoelastic FO properties $C_{\alpha}=\left(c_{\alpha, k j}\right)_{\rightarrow j=1, \ldots, n}^{\downarrow k=1, \ldots, n}$, is linear and proportional by a scalar factor $\kappa_{\alpha}$. This case is formulated as

$C_{\alpha}=\left(c_{\alpha, k j}\right)_{\rightarrow j=1, \ldots, n}^{\downarrow k=1, \ldots, n}=\kappa_{\alpha} C=\kappa_{\alpha}\left(c_{k j}\right)_{\rightarrow j=1, \ldots, n}^{\downarrow k=1, \ldots, n}$ 


\section{a}
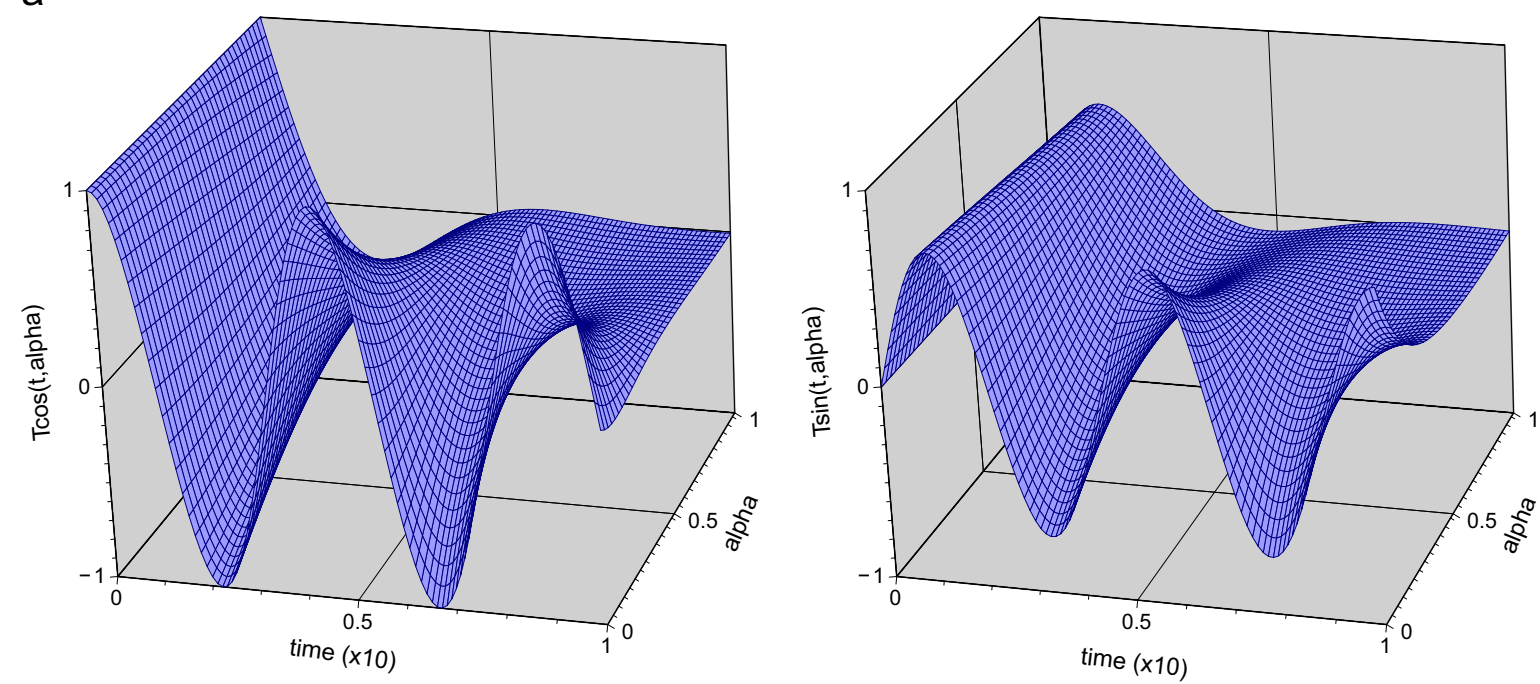

$\mathrm{b}$
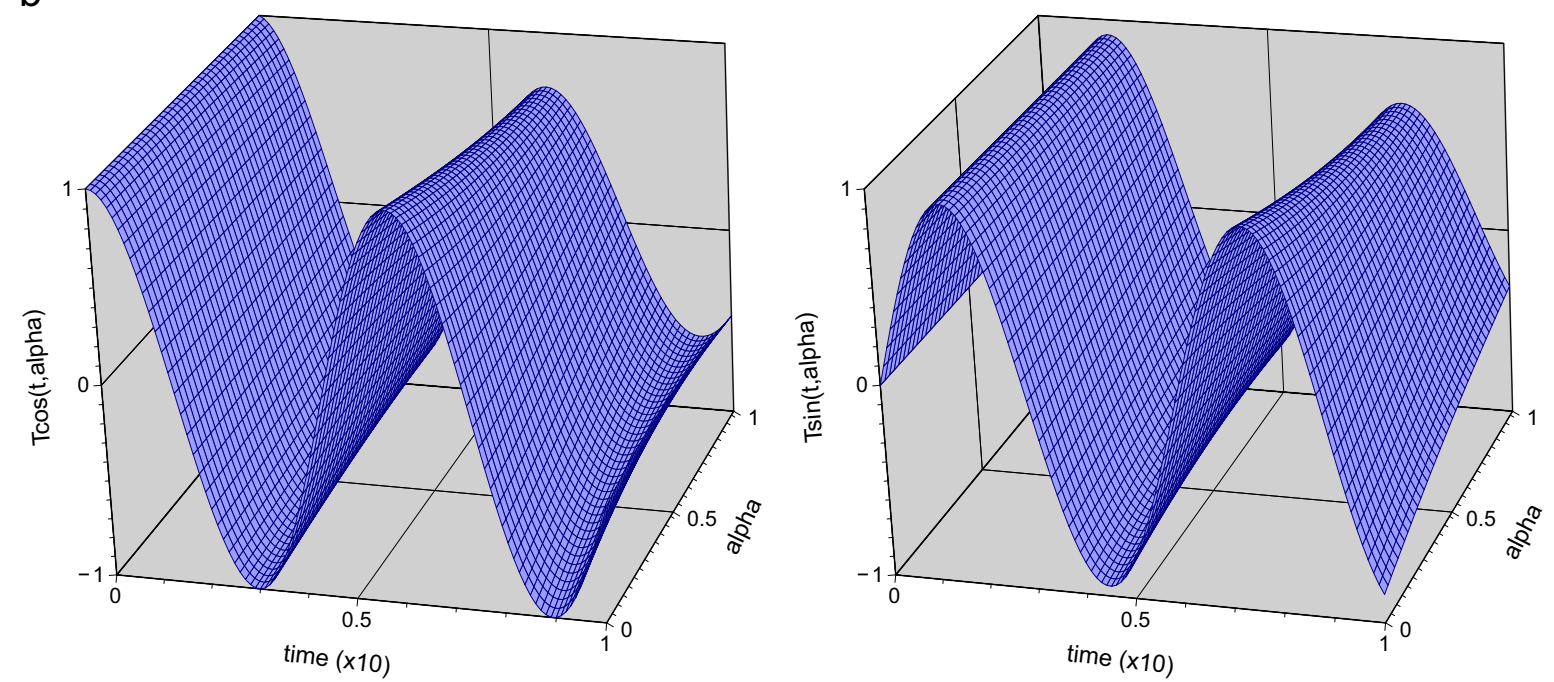

C
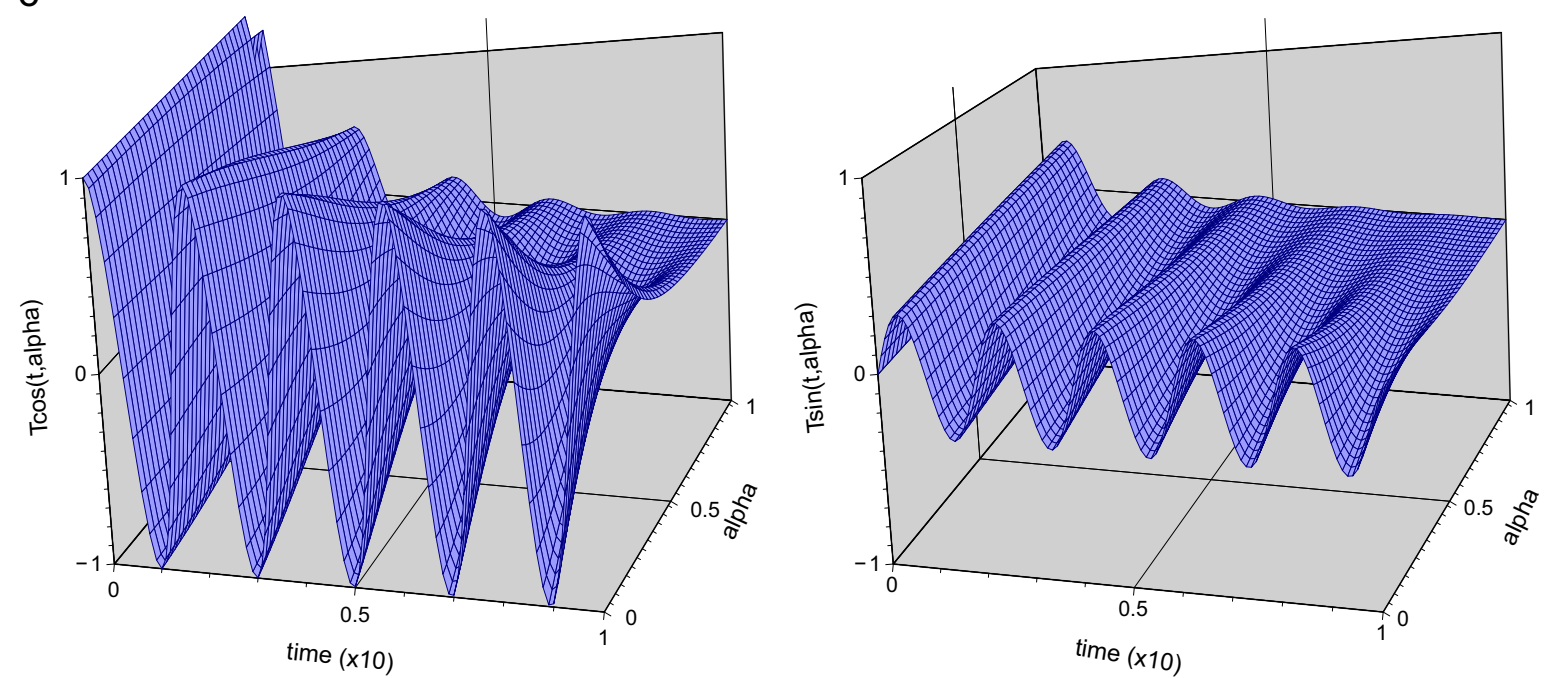

Fig. 1. FO modes $T(t, \alpha)$ for three different kinetic parameters and FO element properties of the system free vibration. (a) $\omega_{(\alpha) s} / \omega_{s}=1$. (b) $\omega_{(\alpha) s} / \omega_{s}=\frac{1}{3}$. (c) $\omega_{(\alpha) s} / \omega_{s}=3$. 


\subsection{Example 1}

A mechanical system with $n$ dof in the form of a mechanical chain satisfying condition (42) and the theorem is adequate for the analysis of FO system vibrations. These conditions are satisfied in $[13,14,29,18,19,21,33]$. Analogies of series, on the basis of analogous mathematical descriptions formulated in the theorem, are identified in kinetic properties [13] of the FO hybrid system vibrations, FO discrete coupled multi-chain system's vibrations [14], main chains and eigen modes of the FO hybrid multi-pendulum system dynamics [17], dynamics of multi-pendulum systems with FO creep elements [18], transfer of signals through hybrid FO homogeneous structure [19], eigen normal modes of eigen time functions of transversal vibrations of creep-connected double plate systems [21], modes of the homogeneous chain dynamics [33], dynamics of coupled FO systems [33], and others.

For all cases [13,14,17-19,21,33], the response of eigen partial FO oscillators along eigen normal coordinates $\zeta_{s}, s=1,2, \ldots, n$, is described by

$\ddot{\zeta}_{s}+\omega_{s}^{2}\left\langle\kappa_{\alpha} D_{t}^{\alpha}\left\{\zeta_{s}\right\}+\zeta_{s}\right\rangle=0, \quad s=1,2, \ldots, n$

where $\omega_{s}^{2}=c_{s s} / a_{s s}$ and $\omega_{(\alpha) s}^{2}=\kappa_{\alpha} \omega_{s}^{2}, s=1,2, \ldots, n$.

A FO system in the form of FO chain with three dof is presented in Fig. 2.

For three particles, of an homogeneous chain system, the set of six characteristic numbers is

$\omega_{1}^{2}=\frac{c}{m}(2-\sqrt{2}), \omega_{(\alpha) 1}^{2}=\kappa_{\alpha} \frac{c}{m}(2-\sqrt{2}), \quad 0<\alpha \leq 1$

$\omega_{2}^{2}=2 \frac{c}{m}, \omega_{(\alpha) 2}^{2}=2 \kappa_{\alpha} \frac{c}{m}, 0<\alpha \leq 1$

$\omega_{3}^{2}=\frac{c}{m}(2+\sqrt{2}), \omega_{(\alpha) 3}^{2}=\kappa_{\alpha} \frac{c}{m}(2+\sqrt{2}), \quad 0<\alpha \leq 1$

\subsection{Example 2}

In [34-38] the dynamics of chain systems with different properties is investigated as separate oscillations, or in hybrid models of Double DNA helix chains, or as a subsystem of mechanical model of oscillations of zona pelucida, before and after fertilization. An analogy between subsystems in the form of homogeneous, or not homogeneous, chains in hybrid biological system's oscillations, is identified and used for explaining its frequency properties. In the cited references, subsystem chain multi-frequency free and forced vibrations are introduced, for frequency analysis of hybrid system different properties, namely in [22], with the eigen main chain modes of the double DNA FO chain helix vibrations, in [34], with the eigen modes of the double DNA chain helix vibrations, in [35], with the modelling oscillations of a zona pelucida, before and after fertilization, and in [36], with the frequency analysis of knot mass particles in an oscillatory spherical net model of the mouse's zona pellucida.

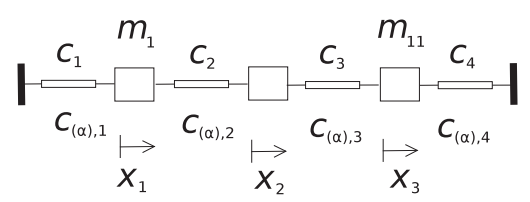

Fig. 2. A FO system in the form of FO chain with three dof.
Phenomenological mappings in vibrations, signals, resonances and dynamical absorptions in chain system dynamics between four special cases of linear ideal elastic systems (in analogy between mechanical chains, tensional systems, discrete continuum chain pendulum system and electrical linear chains system) are considered and presented in Ref. [37]. Using the results [37] of numerical analysis of a eleven particle ideal elastic chain system frequency analysis and changing the springs between the masses lead to the eleventh particle FO chain system, depicted in Fig. 3.

For a FO system, in the form of FO chain with eleven dof that is homogeneous, the series of characteristic numbers of FO system properties are $[23,33,37,38]$

$\omega_{s}^{2}=2 \frac{c}{m}\left(1-\cos \frac{s \pi}{12}\right), \quad s=1, \ldots, 11,0<\alpha \leq 1$

$\omega_{(\alpha) s}^{2}=2 \kappa_{\alpha} \frac{c}{m}\left(1-\cos \frac{s \pi}{12}\right), \quad s=1, \ldots, 11, \quad 0<\alpha \leq 1$

In Fig. 4, the characteristic frequency curve with eleven roots, homogeneous chain system eigen characteristic numbers (squared values of eigen circular frequencies $\omega_{s}^{2}=c_{s s} / a_{s s}$ for free vibrations), is presented.

\section{Qualitative and mathematical analogies between mechanical and electrical FO system vibrations}

In $[39,40]$ Petrović presented the theory of Elements of mathematical phenomenology and Phenomenological mappings. In the last chapter he defined two types of analogies so that results and phenomena in one system could be used for the study of analogous phenomena in other system. Based on these ideas we consider two FO chain system oscillations: a mechanical chain [19] and a electrical ladder [41-43].

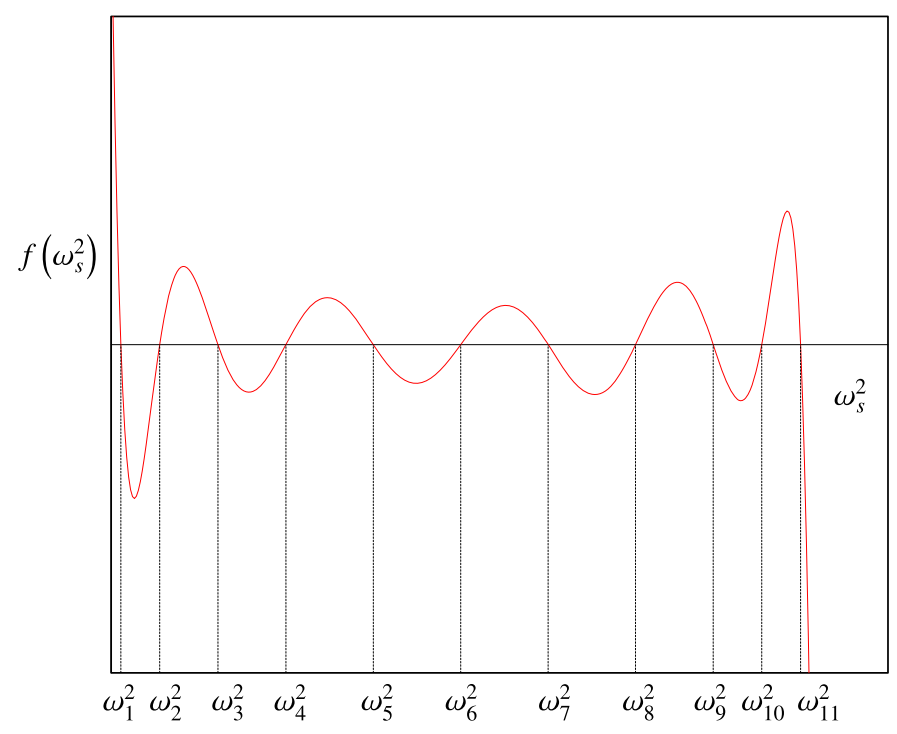

Fig. 4. Characteristic frequency curve with eleven roots, homogeneous chain system eigen characteristic numbers (squared values of eigen circular frequencies $\omega_{s}^{2}=c_{s s} / a_{s s}$ for free vibrations).

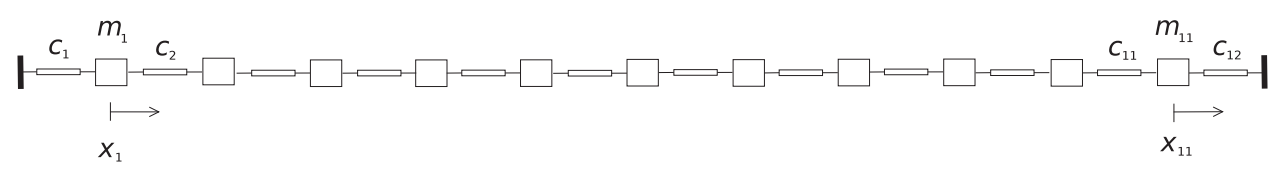

Fig. 3. A FO mechanical chain with eleven dof. 
Table 1

Mathematical analogy between kinetic and material parameters of mechanical and electrical linear systems with one dof.

\begin{tabular}{|c|c|c|c|c|c|c|c|}
\hline & $\begin{array}{l}\text { Linear differential } \\
\text { equations }\end{array}$ & $\begin{array}{l}\text { Generalized system } \\
\text { coordinate }\end{array}$ & $\begin{array}{l}\text { Inertial } \\
\text { coefficient }\end{array}$ & $\begin{array}{l}\text { Elastic rigidity } \\
\text { coefficient }\end{array}$ & $\begin{array}{l}\text { Dissipation } \\
\text { coefficient }\end{array}$ & $\begin{array}{l}\text { External } \\
\text { input }\end{array}$ & $\begin{array}{l}\text { Eigen circular frequency Eigen } \\
\text { resonant frequency }\end{array}$ \\
\hline 1 & $m \ddot{x}+b \dot{x}+c x=F_{0} \cos \Omega t$ & $x$, displacement & $m$, mass & $c$, rigidity & $\begin{array}{l}b \text {, damping force } \\
\text { coefficient }\end{array}$ & $\begin{array}{l}F_{0} \cos \Omega t \\
\text { force }\end{array}$ & $\Omega_{\text {res }}=\omega=\sqrt{\frac{c}{m}}$ \\
\hline 2 & $L \ddot{q}+R \dot{q}+\frac{1}{C} q=V_{0} \cos \Omega t$ & $q$, charge & $L$, inductance & $\frac{1}{C}$, capacitance & $R$, resistance & $\begin{array}{l}V_{0} \cos \Omega t \\
\text { voltage }\end{array}$ & $\Omega_{\text {res }}=\omega=\sqrt{\frac{1}{C L}}$ \\
\hline
\end{tabular}

Table 2

Mathematical analogy between kinetic and material parameters of FO inertia less mechanical visco-elastic and FO electrical resistance elements, $0 \leq \alpha \leq 1$.

\begin{tabular}{|c|c|c|c|c|c|c|}
\hline & $\begin{array}{l}\text { Constitutive relation of the FO } \\
\text { element }\end{array}$ & $\begin{array}{l}\text { Generalized system } \\
\text { coordinate }\end{array}$ & $\begin{array}{l}\text { Inertial } \\
\text { coefficient }\end{array}$ & $\begin{array}{l}\text { Elastic rigidity } \\
\text { coefficient }\end{array}$ & FO coefficients & $\begin{array}{l}\text { FO differential } \\
\text { equations }\end{array}$ \\
\hline 1 & $P(t)=-\left\{c_{0} x(t)+c_{\alpha} D_{t}^{\alpha}[x(t)]\right\}$ & $x$, displacement & $m$, mass & $c_{0}$, rigidity & $\begin{array}{l}c_{(\alpha)} \text {, damping FO force } \\
\text { coefficient }\end{array}$ & $m \ddot{x}+c_{(\alpha)} D_{t}^{\alpha}[x]+c x=0$ \\
\hline 2 & $V(t)=-\left\{\frac{1}{C_{0}} q(t)+R_{\alpha} D_{t}^{\alpha}[q(t)]\right\}$ & $q$, charge & $L$, inductance & $C_{0}$, capacitance & $R_{(\alpha)}$, fractional resistance & $L \ddot{q}+R_{(\alpha)} D_{t}^{\alpha}[q]+\frac{1}{C_{0}} q=0$ \\
\hline
\end{tabular}

Table 3

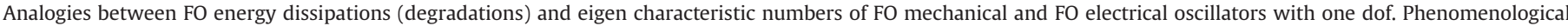
mapping of the corresponding kinetic parameters of eigen FO modes in FO mechanical and FO electrical chains, $0<\alpha \leq 1$.

\begin{tabular}{|c|c|c|c|c|}
\hline & Kinetic energy & Potential energy & $\begin{array}{l}\text { Generalized function of fractional order energy } \\
\text { dissipation }\end{array}$ & $\begin{array}{l}\text { Characteristic numbers for eigen FO normal } \\
\text { modes }\end{array}$ \\
\hline 1 & $\mathbf{E}_{k}=\frac{1}{2} m[\dot{x}(t)]^{2}$, mass & $\begin{array}{l}\mathbf{E}_{p, \alpha}=\frac{1}{2} c_{0}[x(t)]^{2}, \text { elastic } \\
\text { element }\end{array}$ & $\Phi_{\alpha}=\frac{1}{2} c_{\alpha}\left\langle D_{t}^{\alpha}[x(t)]\right\rangle^{2}$ & $\omega_{s}^{2}=\frac{c}{m}, \omega_{(\alpha)}^{2}=\kappa_{\alpha} \frac{c}{m}$ \\
\hline 2 & $\begin{array}{l}\mathbf{E}_{k}=\frac{1}{2} L[\dot{q}(t)]^{2}, \\
\text { inductance }\end{array}$ & $\mathbf{E}_{p, \alpha}=\frac{1}{2} \frac{1}{C_{0}}[q(t)]^{2}$, capacitor & $\Phi_{\alpha}=\frac{1}{2} R_{\alpha}\left\langle D_{t}^{\alpha}[q(t)]\right\rangle^{2}$ & $\omega^{2}=\frac{1}{L C_{0}}, \omega_{(\alpha)}^{2}=\kappa_{\alpha} \frac{1}{L C_{0}}$ \\
\hline
\end{tabular}

Table 4

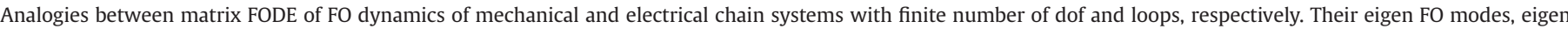

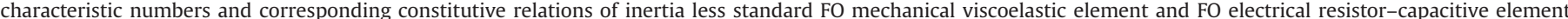
included in the corresponding analogous systems: $0<\alpha \leq 1, s=1, \ldots, n$. Phenomenological mapping between eigen FO modes of mechanical and electrical chains.

\begin{tabular}{|c|c|c|c|c|}
\hline & $\begin{array}{l}\text { Constitutive relation of the FO } \\
\text { element }\end{array}$ & Matrix FODE & $\begin{array}{l}\text { Independent eigen fractional order normal } \\
\text { oscillators }\end{array}$ & Characteristic numbers for eigen FO normal modes \\
\hline 1 & $P(t)=-\left\{c_{0} x(t)+c_{\alpha} D_{t}^{\alpha}[x(t)]\right\}$ & $\mathbf{A}\{\ddot{x}\}+\mathbf{C}_{\alpha}\left\{D_{t}^{\alpha}\{x\}\right\}+\mathbf{C}\{x\}=\{0\}$ & $\ddot{\xi}_{s}+\omega_{(\alpha) s}^{2} D_{t}^{\alpha}\left[\xi_{s}\right]+\omega_{s}^{2} \xi_{s}=0$ & $\begin{array}{l}\omega_{s}^{2}=2 \frac{c}{m}\left(1-\cos \frac{(2 s-1) \pi}{2 n+1}\right) \\
\omega_{(\alpha) s}^{2}=2 \kappa_{\alpha} \frac{c}{m}\left(1-\cos \frac{(2 s-1) \pi}{2 n+1}\right)\end{array}$ \\
\hline 2 & $V(t)=-\left\{\frac{1}{C_{0}} q(t)+R_{\alpha} D_{t}^{\alpha}[q(t)]\right\}$ & $\mathbf{L}\{\ddot{q}\}+\mathbf{R}_{\alpha}\left\{D_{t}^{\alpha}\{q\}\right\}+\mathbf{C}^{*}\{q\}=\{0\}$ & $\ddot{\xi}_{s}+\omega_{(\alpha) s}^{2} D_{t}^{\alpha}\left[\xi_{s}\right]+\omega_{s}^{2} \xi_{s}=0$ & $\begin{array}{l}\omega_{s}^{2}=2 \frac{1}{L C_{0}}\left(1-\cos \frac{(2 s-1) \pi}{2 n+1}\right), \\
\omega_{(\alpha) s}^{2}=2 \kappa_{\alpha} \frac{1}{L C_{0}}\left(1-\cos \frac{(2 s-1) \pi}{2 n+1}\right)\end{array}$ \\
\hline
\end{tabular}

Table 5

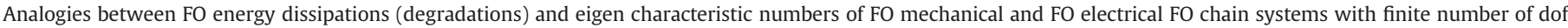
and loops, respectively. Phenomenological mapping of the corresponding kinetic parameters of eigen FO modes, $0<\alpha \leq 1, \eta_{\mathrm{s}}, s=1, \ldots, n$.

\begin{tabular}{|c|c|c|c|c|}
\hline & Kinetic energy & Potential energy & Generalized function of fractional order energy dissipation & Characteristic numbers for eigen FO normal modes \\
\hline \multirow[t]{2}{*}{1} & $2 \mathbf{E}_{k}=(\dot{x}) \mathbf{A}\{\dot{x}\}$ & $2 \mathbf{E}_{p}=(x) C\{x\}$ & $2 \mathbf{P}_{\alpha \neq 0}=\left(D_{t}^{\alpha}\{x\}\right) C_{\alpha}\left\{D_{t}^{\alpha}\{x\}\right\}$ & $\omega_{s}^{2}=2 \frac{c}{m}\left(1-\cos \frac{(2 s-1) \pi}{2 n+1}\right)$ \\
\hline & $2 \mathbf{E}_{k}=\sum_{s=1}^{s=n} \dot{\eta}_{s}^{2}$ & $2 \mathbf{E}_{p}=\sum_{s=1}^{s=n} \omega_{s}^{2} \eta_{s}^{2}$ & $2 \mathbf{P}_{\alpha}=\sum_{s=1}^{s=n} \omega_{(\alpha), s}^{2}\left(D_{t}^{\alpha}\left[\eta_{s}\right]\right)^{2}$ & $\omega_{(\alpha) s}^{2}=2 \kappa_{\alpha} \frac{c}{m}\left(1-\cos \frac{(2 s-1) \pi}{2 n+1}\right)$ \\
\hline \multirow[t]{2}{*}{2} & $2 \mathbf{E}_{k}=(\dot{q}) \mathbf{L}\{\dot{q}\}$ & $2 \mathbf{E}_{p}=(q) \mathbf{C}^{*}\{q\}$ & $2 P_{\alpha \neq 0}=\left(D_{t}^{\alpha}\{q\}\right) \mathbf{R}_{\alpha}\left\{D_{t}^{\alpha}\{q\}\right\}$ & $\omega_{s}^{2}=2 \frac{1}{L C_{0}}\left(1-\cos \frac{(2 s-1) \pi}{2 n+1}\right)$ \\
\hline & $2 \mathbf{E}_{k}=\sum_{s=1}^{s=n} \dot{\eta}_{s}^{2}$ & $2 \mathbf{E}_{p}=\sum_{s=1}^{s=n} \omega_{s}^{2} \eta_{s}^{2}$ & $2 \mathbf{P}_{\alpha}=\sum_{s=1}^{s=n} \omega_{(\alpha), s}^{2}\left(D_{t}^{\alpha}\left[\eta_{s}\right]\right)^{2}$ & $\omega_{((\alpha) s}^{2}=2 \kappa_{\alpha} \frac{1}{L C_{0}}\left(1-\cos \frac{(2 s-1) \pi}{2 n+1}\right)$ \\
\hline
\end{tabular}




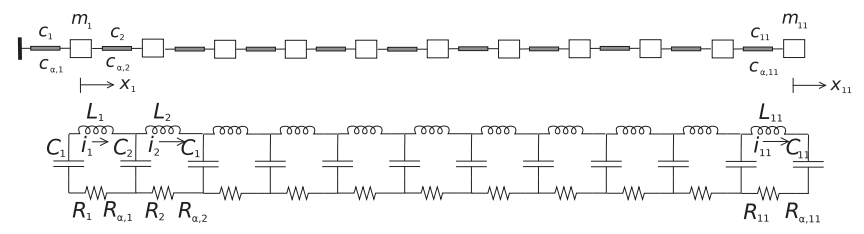

Fig. 5. Two analogous FO system oscillations: mechanical chain fixed at left end and with eleven dof and electrical circuit with eleven coupled loops.

In Tables 1-5 we present the analogous parameters of these systems.

Let us consider vibrations in the two FO systems represented in Fig. 5. These two analogous system oscillations are

- a FO mechanical chain, fixed at left end and with 11 dof;

- a FO electrical network with 11 coupled loops.

Between these two systems it is possible to establish an analogy of the FO vibrations, normal FO modes and eigen normal coordinates.

Let us note the difference between $\eta_{s}, s=1, \ldots, n$, eigen normal coordinates (modes) and $\xi_{s}, s=1, \ldots, n$, eigen main coordinates (modes).

Transformations $\mathcal{A}=\mathbf{R}^{\prime} \mathbf{A R}=\left(a_{s s}\right)$ and $\mathcal{C}=\mathbf{R}^{\prime} C \mathbf{R}=\left(c_{s s}\right)$, by means of modal matrix $\mathbf{R}$ defined in expressions (25) and (26), are diagonal matrices. With the modal matrix $\mathbf{R}$, the generalized coordinates are expressed by eigen main coordinates $\xi_{s}$ in the form: $\{x\}=\sum_{s=1}^{s=n}\left\{K_{n k}^{s}\right\} C_{s} \cos \left(\omega_{s} t+\beta_{s}\right)=\mathbf{R}\left\{\xi_{s}\right\}$. The normalized modal matrix $\mathbf{V}$ is in the form: $\mathbf{V}=\left(\left\{D_{s} K_{n k}^{S}\right\}\right)=\left(D_{s} K_{n k}^{S}\right)_{\rightarrow s=1, \ldots,{ }_{n}}^{\downarrow k}$, with normalizations: $\mathbf{V}^{\prime} \mathbf{A V}=\mathbf{I} \Rightarrow D_{s}=\cdots$.

By means of the normalized modal matrix $\mathbf{V}$, the generalized coordinates are expressed by eigen normal coordinates $\eta_{s}$ in the form: $\{x\}=\sum_{s=1}^{s=n_{1}}\left\{D_{s} K_{n k}^{s}\right\} \tilde{C}_{s} \cos \left(\omega_{s} t+\beta_{s}\right)=\mathbf{V}\left\{\eta_{s}\right\}$.

The transformation of the matrix of inertia coefficients in eigen normal coordinates $\eta_{\mathrm{s}}$ produces a diagonal with unique elements $\mathbf{I}=\mathbf{V}^{\prime} \mathbf{A} \mathbf{V}$ and the transformation of matrix of coefficients of system rigidity in eigen normal coordinates $\eta_{s}$ produces the corresponding diagonal with squared eigen circular frequencies elements $\boldsymbol{\Lambda}=\mathbf{V}^{\prime} \mathbf{C} \mathbf{V}=\operatorname{diag}\left(\omega_{1}^{2}, \omega_{2}^{2}, \ldots, \omega_{n}^{2}\right)$

Mechanical system: A FO element of negligible mass is in the form of an axially stressed rod without bending. This element has the ability to resist to deformation under static and dynamic conditions. A FO creep element, for which the constitutive stressstrain relation for the restitution force is function of element elongation, is given by an expression containing a term in the form of FO derivative. This constitutive relation follows (1) and (2).

For $0<\alpha<1$, the constitutive relation turns to the form $P(t)=-\left\{c_{0} x(t)+c_{\alpha} \mathrm{D}_{t}^{\alpha}[x(t)]\right\}$, the properties of element are viscoelastic fractional order. Under dynamic loading, the element has potential energy $\mathbf{E}_{p, \alpha}=\frac{1}{2} c_{0}[x(t)]^{2}$ and dissipation of element energy is proportional to the FO derivative of the coordinate deformation. Also, Rayleigh's function of dissipation of a non-conservative FO generalized is in the form: $\Phi_{\alpha}=\frac{1}{2} c_{\alpha}\left\langle D_{t}^{\alpha}[x(t)]\right\rangle^{2}$.

Electrical system: A FO capacitive-resistive element is possible to define by means of the following constitutive relation:

$v(t)=-\left\{\frac{1}{C_{0}} q(t)+R_{\alpha} D_{t}^{\alpha}[q(t)]\right\}$

where $v(t)$ denotes electrical voltage, $q(t)$ charge, $i(t)=\dot{q}(t)$ is the electric current, or $q(t)=\int_{0}^{t} i(t) d t$, and $R_{\alpha}$ represents the coefficient of FO dissipation thermal energy in the FO dissipative element, with $0<\alpha \leq 1$.

For $\alpha=0$ it yields

$v(t)=-\left\{\frac{1}{C_{0}}+R_{\alpha=0}\right\} q(t)=\left\langle\frac{1}{C_{0}}+\frac{1}{C_{\alpha=0}}\right\rangle q(t)$,

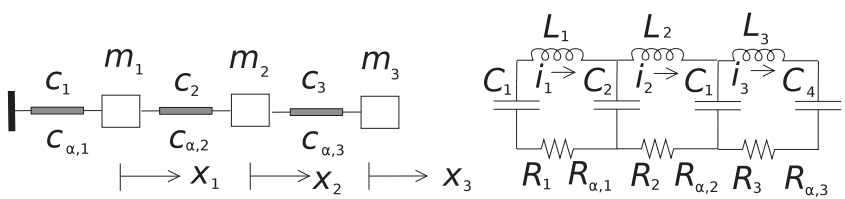

Fig. 6. Two analogous FO system oscillations with three dof: FO mechanical chain fixed at left end and FO electrical circuit.

and for $\alpha=1$ it yields

$v(t)=-\left\{\frac{1}{C_{0}} q(t)+R_{\alpha=1} \dot{q}(t)\right\}$.

Therefore, we obtain $R_{\alpha=0}=1 / C_{\alpha=0}$ or $C_{\alpha=0}=1 / R_{\alpha=0}$.

If the systems of Fig. 5 represent two analogous homogeneous FO oscillation systems, with eleven dof, then the eigen characteristic numbers express FO properties $[23,24]$. For FO mechanical system:

$\omega_{s}^{2}=2 \frac{c}{m}\left(1-\cos \frac{(2 s-1) \pi}{23}\right)$

$\omega_{(\alpha) s}^{2}=2 \kappa_{\alpha} \frac{c}{m}\left(1-\cos \frac{(2 s-1) \pi}{23}\right)$

where $s=1,2,3, \ldots, 11, \kappa_{\alpha}=c_{\alpha} / c$ and $0<\alpha \leq 1$.

For a FO electrical system:

$\omega_{s}^{2}=2 \frac{1}{L C_{0}}\left(1-\cos \frac{(2 s-1) \pi}{23}\right)$

$\omega_{(\alpha) s}^{2}=2 \kappa_{\alpha} \frac{1}{L C_{0}}\left(1-\cos \frac{(2 s-1) \pi}{23}\right)$

where $s=1,2,3, \ldots, 11, \kappa_{\alpha}=R_{\alpha} / C_{0}$ and $0<\alpha \leq 1$.

If systems at Fig. 6 are two analogous homogeneous FO system oscillations with 3 dof, then $[23,24]$ for both systems, the eigen characteristic numbers expressing FO properties of the system are Mechanical system

$\omega_{s}^{2}=2 \frac{c}{m}\left(1-\cos \frac{(2 s-1) \pi}{7}\right)$

$\omega_{(\alpha) s}^{2}=2 \kappa_{\alpha} \frac{c}{m}\left(1-\cos \frac{(2 s-1) \pi}{7}\right)$

where $s=1,2,3, \ldots, 11, \kappa_{\alpha}=c_{\alpha} / c$ and $0<\alpha \leq 1$.

Electrical system

$\omega_{s}^{2}=2 \frac{1}{L C_{0}}\left(1-\cos \frac{(2 s-1) \pi}{7}\right)$

$\omega_{(\alpha) s}^{2}=2 \kappa_{\alpha} \frac{1}{L C_{0}}\left(1-\cos \frac{(2 s-1) \pi}{7}\right)$

where $s=1,2,3, \kappa_{\alpha}=R_{\alpha} / C_{0}$ and $0<\alpha \leq 1$

\section{Concluding remarks}

For investigating FO system dynamics it is important to determine the kinetic and frequency characteristics of the corresponding linear system, with eigen main and eigen normal coordinates, as a basis for FODE transformation and to analyze the normal FO modes for different cases. Only in a special case exists a family of FO system oscillation with independent normal FO modes. For that family of systems, a theorem is defined and demonstrated.

Mihailo Petrovic's theory of mathematical phenomenology elements [39,40,37], phenomenological mapping and mathematical analogies, represents an useful tool for integrating the knowledge obtained in distinct scientific areas on the basis of 
phenomenological mappings, analogous models of the FO system's dynamics of different nature (mechanical, electrical, biomechanical, physico-chemical, socio-economical).

We consider transversal vibrations of an eleven deformable beam hybrid FO system on a discrete continuum FO layer, with described by eleven coupled partial FODE along the beam's transversal displacements $w_{k}(x, t), k=1, \ldots, 11$. Two examples, for transversal vibrations of an eleven deformable plate and transversal vibrations of an eleven deformable membrane hybrid FO systems, on a discrete continuum FO layer, are considered. For solving the coupled partial differential equations, we can use similar series along the eigen amplitude functions, the frequency time functions, $T_{k(n)}(t)$, for eleven beam system and $T_{k(n m)}(t)$, $k=1, \ldots, 11, n, m=1, \ldots \infty$, for eleven plate and eleven membrane systems. Then phenomenological mapping and the mathematical analogy between eigen time functions, corresponding to eigen amplitude functions, is possible to use.

Through the use of phenomenological mapping, data obtained for free FO vibrations can be used for a qualitative explanation of the characteristic time functions of transversal vibration of coupled multi-deformable body hybrid FO systems.

The results presented here are applicable to biological chain FO oscillators, such as a FO model of the DNA double helix [21,34,35]. The discrete continuum method $[13,14,17-19,44]$ is based on coupled FO chains as a modelling abstraction of the real continuum, resulting in the discretization of structures. The results can be applied to models obtained by discrete continuum method for solving the dynamics of FO systems of different kinds.

It is used as a mathematical description of a chain mechanical system with a finite number of mass particles coupled by standard light FO elements and finite number of dof expressed by the corresponding generalized independent coordinates, translating displacements and the corresponding analysis of solutions. For free vibrations, series of similar multi-frequency FO regimes are identified. Adopting a mathematical analogy and phenomenological mappings it analyzed the dynamics of series and other chainlike models.

This paper consists of the second study from a series of four works related to FO chain system dynamics and eigen FO modes, free and forced chain non-linear dynamics, eigen main chains in complex coupled chains in the models abstraction of the real system bio-dynamics and energy analysis of model with coupled chains. We should point out comprehensive review papers [45-47] written by Rosikin and Shitikova, containing review and results achieved in the field of applying FO derivatives in mechanics.

\section{Acknowledgments}

Parts of this research were supported by the Ministry of Sciences of Republic Serbia through Mathematical Institute SANU Belgrade Grants OI 174001" Dynamics of hybrid systems with complex structures. Mechanics of materials", Faculty of Mechanical Engineering University of Niš.

\section{References}

[1] K. Oldham, J. Spanier, The Fractional Calculus: Theory and Application of Differentiation and Integration to Arbitrary Order, Academic Press, New York, 1974

[2] S. Samko, A. Kilbas, O. Marichev, Fractional Integrals and Derivatives: Theory and Applications, Gordon and Breach Science Publishers, Amsterdam, 1993.

[3] K. Miller, B. Ross, An Introduction to the Fractional Calculus and Fractional Differential Equations, John Wiley and Sons, New York, 1993.

[4] I. Podlubny, Fractional Differential Equations, Volume 198: An Introduction to Fractional Derivatives, Fractional Differential Equations, to Methods of Their Solution, Mathematics in Science and Engineering, Academic Press, San Diego, 1998.
[5] A. Kilbas, H. Srivastava, J. Trujillo, Theory and Applications of Fractional Differential Equations, vol. 204, North-Holland Mathematics Studies, Elsevier, Amsterdam, 2006.

[6] G. Zaslavsky, Hamiltonian Chaos and Fractional Dynamics, Oxford University Press, Oxford, 2005.

[7] J. Sabatier, O.P. Agrawal, J.T. Machado, Advances in Fractional Calculus: Theoretical Developments and Applications in Physics and Engineering, Springer, Dordrecht, The Netherlands, 2007.

[8] V. Tarasov, Fractional Dynamics: Applications of Fractional Calculus to Dynamics of Particles, Fields and Media, Springer, New York, 2010.

[9] G.-C. Wu, D. Baleanu, Chaos synchronization of the discrete fractional logistic map, Signal Process. 102 (2014) 96-99.

[10] G.-C. Wu, D. Baleanu, S.-D. Zeng, Discrete chaos in fractional sine and standard maps, Phys. Lett. A 378 (5-6) (2014) 484-487.

[11] G.-C. Wu, D. Baleanu, Discrete fractional logistic map and its chaos, Nonlinear Dyn. 75 (1-2) (2014) 283-287.

[12] O.A. Goroško, K.R. (Stevanović) Hedrih, Analitička dinamika (mehanika) diskretnih naslednih sistema, Analytical Dynamics (Mechanics) of Discrete Hereditary Systems, University of Niš, Niš, Servia, 2001.

[13] K.R. (Stevanović) Hedrih, The fractional order hybrid system vibrations, in: Advances in Nonlinear Sciences, ANN, Belgrade, Servia, 2008.

[14] K.R. (Stevanović) Hedrih, Analytical mechanics of fractional order discrete system vibrations, in: Advances in Nonlinear Sciences, JANN, Belgrade, Servia, 2011.

[15] M. Enelund, Fractional Calculus and Linear Viscoelasticity in Structural Dynamics (Ph.D. thesis), Chalmers University of Technology, Göteborg, Sweden, 1996.

[16] R. Gorenflo, F. Mainardi, Fractional calculus, integral and differential equations of fractional order, in: CISM Lecture Notes, 2000, pp. 223-276 (preprint).

[17] K.R. (Stevanović) Hedrih, Main chains and eigen modes of fractional order hybrid multipendulum system dynamics, Phys. Scr. 2009 (T136) (2009) 014038. http://dx.doi.org/10.1088/0031-8949/2009/T136/014038.

[18] K.R. (Stevanović) Hedrih, Dynamics of multi-pendulum systems with fractional order creep elements, J. Theor. Appl. Mech. 46 (3) (2009) 483-509.

[19] K.R. (Stevanović) Hedrih, Considering transfer of signals through hybrid fractional order homogeneous structure, in: G. Dimirovski (Ed.), Proceedings of Selected AAS 2009 Papers, Skopje, Istambul, 2009, pp. 19-24, Keynote Lecture.

[20] F. Mainardi, Fractional Calculus and Waves in Linear Viscoelasticity: An Introduction to Mathematical Models, Imperial College Press, London, 2010.

[21] K.R. (Stevanović) Hedrih, Partial fractional order differential equations of transversal vibrations of creep-connected double plate systems, in: A. L. Mahauté, J.A.T. Machado, J.C. Trigeassou, J. Sabatier (Eds.), Fractional Differentiation and its Applications, UBooks, Germany, 2005, pp. 289-302.

[22] K.R. (Stevanović) Hedrih, A.N. Hedrih, Eigen main chain modes of the double DNA fractional order chain helix vibrations (part I), in: Proceedings of Second International Congress of Serbian Society of Mechanics-IConSSM 2009, Palić, Subotica, 2009, pp. 1-15.

[23] D. Rašković, Teorija oscilacija (Theory of Oscillations), Naučna knjiga, 1952.

[24] D. Rašković, Analitička mehanika (Analytical Mechanics), Mašinski fakultet Kragujevac, 1974.

[25] R.W. Clough, J. Penzien, Dynamics of Structures, McGraw-Hill, New York, 1975

[26] I. Schäfer, S. Kempfle, Impulse responses of fractional damped systems, Nonlinear Dyn. 38 (1-4) (1997) 61-68.

[27] Y.A. Rossikhin, M.V. Shitikova, Application of fractional calculus for dynamic problems of solid mechanics: novel trends and recent results, Appl. Mech. Rev. 63 (1) (2010) 010801.

[28] S. Timoshenko, Vibration Problems in Engineering, 1st Edition, Van Nostrand, New York, 1928.

[29] B.S. Baćlić, T. Atanacković, Stability and creep of a fractional derivative order viscoelastic rod, Bulletin T, CXXI de L'Academie Serbe des Sciences st de Arts, Class des Sciences Mathematques et Naturelles Sciences Mathematiques 25 (2000) 115-131.

[30] K.R. (Stevanović) Hedrih, Longitudinal vibration of a fractional derivative order rheological rod with variable cross section, Facta Univ. Ser.: Mech. Autom. Control Robot. 3 (12) (2002) 327-350.

[31] L. Suarez, A. Shokooh, Response of systems with damping materials modeled using fractional calculus, Appl. Mech. Rev. 48 (11S) (1995) S118-S126.

[32] Y.A. Rossikhin, M.V. Shitikova, Application of fractional derivatives to the analysis of damped vibrations of viscoelastic single mass systems, Acta Mech. $120(1-4)(1997)$ 109-125.

[33] K.R. (Stevanović) Hedrih, Modes of the homogeneous chain dynamics, Signal Process. 86 (10) (2006) 2678-2702.

[34] K.R. (Stevanović) Hedrih, A.N. Hedrih, Eigen modes of the double DNA chain helix vibrations, J. Theor. Appl. Mech. 1 (48) (2010) 219-231.

[35] A.N. Hedrih, Modeling oscillations of zona pelucida before and after fertilization, EUROMECH Newsl. 40 (2011) 6-14.

[36] A.N. Hedrih, Frequency analysis of knot mass particles in oscillatory spherical net model of mouse zona pellucida, in: Abstract book of 23rd International Congress of Theoretical and Applied Mechanics, Beijing, China, 2012, p. 209.

[37] K.R. (Stevanović) Hedrih, A.N. Hedrih, Phenomenological mapping and dynamical absorptions in chain systems with multiple degrees of freedom. J. Vib. Control, 2014, http://dx.doi.org/10.1177/1077546314525984.

[38] K.R. (Stevanović) Hedrih, Dynamics of coupled systems, Nonlinear Anal.: Hybrid Syst. 2 (2) (2008) 310-334.

[39] M. Petrović, Elementi matematičke fenomenologije (Elements of mathematical phenomenology), Srpska kraljevska akademija, Beograd, Serbia, 1911. 
[40] M. Petrović, Fenomenološko Preslikavanje (Phenomenological Map), Srpska kraljevska akademija, Beograd, Serbia, 1933.

[41] J.A.T. Machado, Fractional generalization of memristor and higher order elements, Commun. Nonlinear Sci. Numer. Simul. 18 (12) (2013) 264-275.

[42] J.A.T. Machado, A fractional approach to the Fermi-Pasta-Ulam problem, Eur. Phys. J. Special Top. 222 (8) (2013) 1795-1803.

[43] J.A.T. Machado, Dynamics of a backlash chain, Cent. Eur. J. Phys. 11 (10) (2013) 1268-1274.

[44] K.R. (Stevanović) Hedrih, A.N. Hedrih, Considering vibrations of the double DNA main chains via a model with hereditary properties, in: Proceedings $2 \mathrm{nd}$ International Congress of Serbian Society of Mechanics-IConSSM 2009, The
International Federation of Automatic Control, Ohrid, Republic of Macedonia, 2009, pp. 341-346.

[45] Y.A. Rossikhin, M.V. Shitikova, Application of fractional calculus for dynamic problem of solid mechanics: novel trends and resent results, Appl. Mech. Rev. 63 (1) (2009) 010801-1-010801-52.

[46] Y.A. Rossikhin, M.V. Shitikova, Applications of fractional calculus to dynamic problems of linear and nonlinear hereditary mechanics of solids, Appl. Mech. Rev. 50 (1) (1997) 15-67.

[47] Y.A. Rossikhin, Reflections on two parallel ways in the progress of fractional calculus in mechanics of solids, Appl. Mech. Rev. 63 (1) (2010) 010701-1010701-12. 